\title{
Interaction between a Flat Plate and a Circular Subsonic Jet
}

\author{
J. L. T. Lawrence ${ }^{1}$, M. Azarpeyvand ${ }^{2}$ and R. H. Self ${ }^{3}$ \\ Institute of Sound and Vibration Research, University of Southampton, Southampton, Hampshire, SO17 1BJ, UK
}

This paper reports an extensive near- and far-field analysis of the noise generated by an isothermal, subsonic, circular jet in the presence of a solid, flat plate shield. Far-field polar and azimuthal acoustic images are presented initially to characterize the interaction noise source. Near-field streamwise microphone phase analysis along the plate trailing edge reveals a deeper understanding of the link between the jet hydrodynamic field (both linear and non-linear regions) and the mechanisms behind interaction noise generation. Near-field point spectrum data have also been used successfully to validate Amiet's far-field trailing edge dipole prediction code for low-speed jet acoustic Mach numbers.

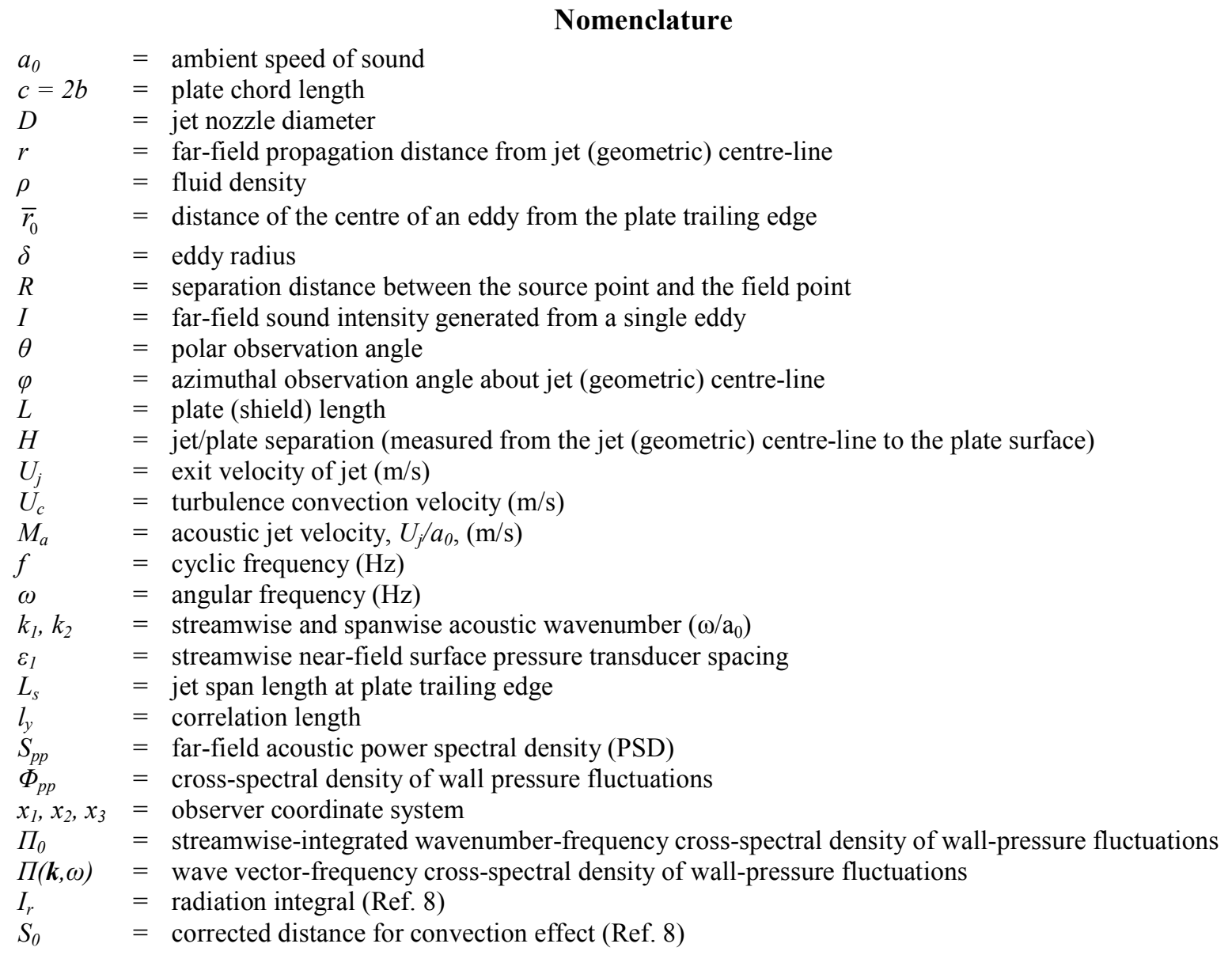

\footnotetext{
${ }^{1}$ Research Engineer, Fluid Dynamics and Acoustics Group, ISVR, Student Member AIAA

${ }^{2}$ Research Fellow, Fluid Dynamics and Acoustics Group, ISVR

${ }^{3}$ Senior Lecturer, Fluid Dynamics and Acoustics Group, ISVR, Member AIAA
} 


\section{Introduction}

Current research into jet installation noise is being fuelled by the demand for more powerful and efficient engines. Thus, as under-wing-mounted engine diameters increase, jet axes must move closer to the airframe in order to maintain the same ground clearance. Together with the ever-increasingly challenging noise requirements for tomorrow's civilian aircraft, this close-coupling now means that installation noise plays a major part in sideline, flyover and approach noise certification measurements. Before attempting to predict the complex interactions between a coaxial, heated jet in-flight beneath a 3D lifting wing and flap, it is first necessary to examine and understand a more fundamental setup. Only then can one attempt to isolate and link specific, geometric parameters of the wing to, say, the turbulence properties of the local shear layer within the jet.

Previous studies ${ }^{1-8}$ have suggested that the most dominant source of interaction noise is generated by the turbulence within the free shear layer of the jet convecting downstream past the trailing edge (TE) of the wing or flap. There are potentially two noise generating mechanisms at work here. The first is due to unsteady loadings on the wing surface, which produce dipolar noise, as per Curle's formulation for acoustically compact surfaces ${ }^{1}$. The second mechanism, as first discovered by Ffowcs-Williams and Hall, comes as a result of diffraction of the jet's hydrodynamic near-field about the $\mathrm{TE}^{2}$. However, since both of these mechanisms result from convecting turbulence, their respective radiation frequency content is inherently similar and, hence, challenging to separate. Generically, interaction noise is seen as a low frequency augmentation above the isolated jet noise, see Figure 1.

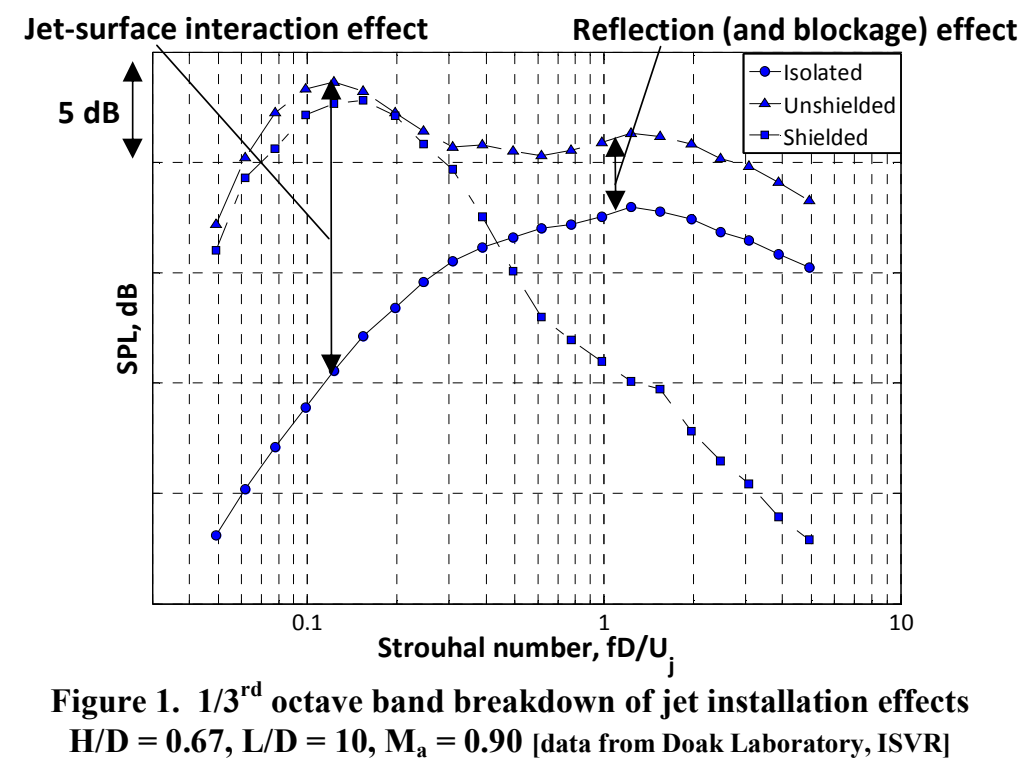

This paper will predominantly focus on this augmentation effect while building upon Head and Fisher's previous study on jet/surface interaction noise ${ }^{4}$. In 1976, Head \& Fisher presented a series of acoustic experiment results involving a cold, subsonic jet in the presence of a solid shield. It was suggested that the additional low frequency noise was due to a dipole source driven by local near-field acoustic pressures at the shield TE. However, when used to inform future aircraft design ventures, two crucial short-comings have been identified. Firstly, the smallest jetshield separation distance $(H)$ investigated was $2 \mathrm{D}$. Interest today, however, is focused upon separation distances well below this value, where the interaction physics of the diffraction of the jet's acoustic, linear hydrodynamic and non-linear hydrodynamic fields is, as yet, not entirely understood. Secondly, confident and robust hypotheses concerning the source generation mechanisms and directivity patterns were difficult to make due to the sparselypopulated near- and far-field microphone arrays, especially into the forward far-field jet arc. Thus, the principle goal of this particular study is to further the investigation of close-coupled under-wing-mounted jets with a view to predicting the far-field noise.

The layout of the paper is as follows. Initially presented are the far-field acoustic results from an experimental test campaign conducted in the Anechoic Doak Laboratory, at the Institute of Sound and Vibration, within the University of Southampton, UK (ISVR). The experiment essentially consisted of a cold, conical jet in the presence of a solid, flat plate under static ambient flow conditions. The plate was positioned on a traversable arm and at a 
variety of axial and radial locations near the jet for a selection of subsonic jet acoustic Mach numbers $\left(\mathrm{M}_{\mathrm{a}}=0.3,0.5\right.$, 0.75 and 0.9). Acoustic measurements from both near- and far-field microphone arrays were made. Presented in this paper are the results from jet-plate configurations including: $\mathrm{H} / \mathrm{D}=0.67,1,2$ and $\mathrm{L} / \mathrm{D}=2,4,7,10$. A detailed analysis and discussion of the behaviour of the TE "dipole" source is presented followed by additional information concerning both the linear and non-linear hydrodynamic near-fields of the jet.

\section{Experimental Setup}

\section{A. Facility and Test Apparatus}

The Anechoic Doak Laboratory facility (Figure 2 ) is approximately $15 \mathrm{~m} \times 7 \mathrm{~m} \times 5 \mathrm{~m}$ high and is fully anechoic down to $400 \mathrm{~Hz}$. The maximum achievable acquisition frequency is $40 \mathrm{kHz}$. For these jet interaction experiments, the airflow to the test nozzle passed through an upright labyrinth silencer into the jet-pipe. The cold, subsonic jet was operated from a $38.1 \mathrm{~mm}$ exit-diameter, convergent conical nozzle in the presence of a flat plate wing over a range of fully expanded jet velocities. This nozzle was chosen because its aerodynamic and acoustic characteristics were well-documented in the Noise Test Facility (NTF) at QinetiQ, Farnborough, UK. The $6 \mathrm{~mm}$ thick aluminum alloy plate was machined down to a $1 \mathrm{~mm}$ trailing edge.

The conditions of the air stream and acoustic Mach number set points were determined from

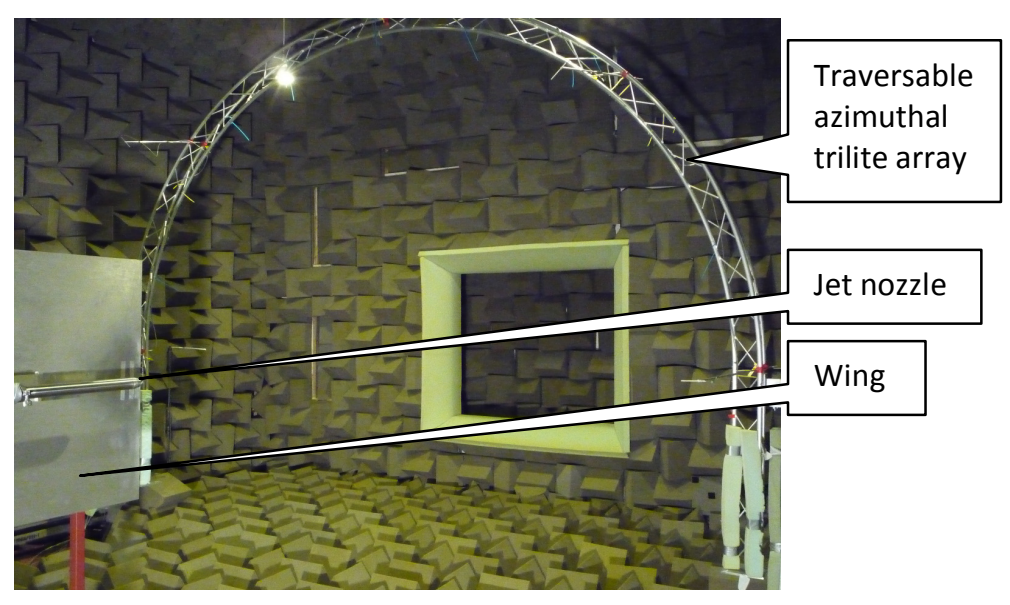

Figure 2. Doak Laboratory far-field experiment setup measurements of total temperature and total pressure using a thermocouple probe and Pitot probe installed well upstream of the nozzle. To ensure accurate acoustic propagation representation, ambient temperature and pressure instrumentation was also setup within the laboratory.

Since the jet and wing system are the main components affecting the noise characteristics for an under-wing engine, the dimensions of these components and the range of values of the installation parameters were carefully chosen to represent a generic, medium range, civilian transport aircraft. In addition to the flat plate configurations, plate plus flap installations were also tested. This paper, however, only deals with the flat plate results.

\section{B. Instrumentation and Procedures}

\section{Far-field setup}

Five 1/4-in. condenser microphones populated a far-field azimuthal traversable array including azimuthal observation angles $(\varphi) 0^{\circ}-180^{\circ}$ at $45^{\circ}$ intervals (Figure $3 \mathrm{~b}$ ). These microphones on the traversable array were positioned at $\mathrm{r}=2.02 \mathrm{~m}(\approx 53 \mathrm{D})$ from the jet nozzle exit in the geometric far-field, at the closest polar observation angle $(\theta)$ of $90^{\circ}$ (Figure 3a). The azimuthal array was traversed up and down the laboratory to incorporate polar observation angles $(\theta) 130^{\circ}-60^{\circ}$ in $10^{\circ}$ intervals. Additional fixed polar observation microphones were positioned at $\theta=50^{\circ}$ (at $\varphi=0^{\circ}$ and $180^{\circ}$ ) and at $\theta=40^{\circ}$ (at $\varphi=0^{\circ}$ and $180^{\circ}$ ) to complete the 3D sound field map.

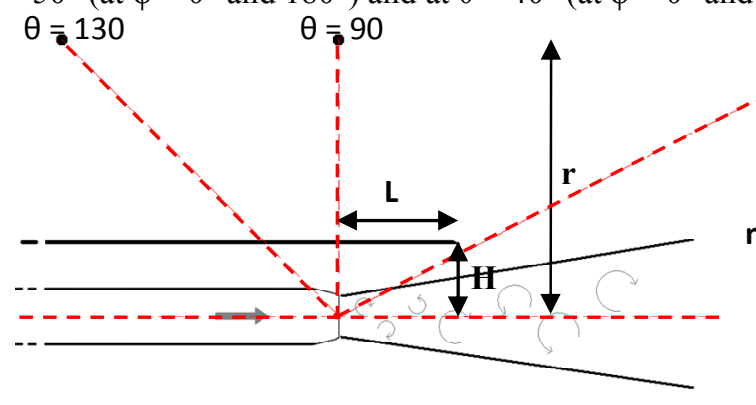

(a)

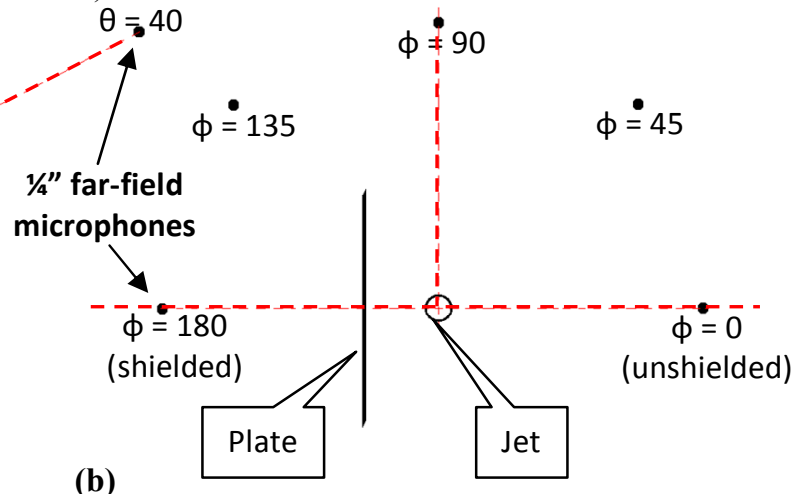

(b)

Figure 3. (a) Polar arrangement of microphones (plan view), (b) azimuthal arrangement of microphones on the traversable trilite array (jet axis view)

3

American Institute of Aeronautics and Astronautics 


\section{Near-field setup}

Fourteen phase-matched near-field pressure transducers were positioned flush to the surface of the plate along the streamwise and spanwise axes (see Figure 4) in order to track the velocity and strength of three jet pressure fields as they each are convected pass the plate TE. These three fields include: 1) the jet acoustic field (which will include contributions from both quadrupole mixing noise and dipole TE noise sources); 2) the rotational, non-linear hydrodynamic pressure field (i.e. the quasi-orderly convecting eddies) and 3) the irrotational, linear hydrodynamic pressure field. It is possible to separate these fields with an understanding of the different wave propagation speeds involved. In order to filter the acoustic field from the hydrodynamic fields, for example, one must choose the supersonically convecting wavenumbers. This analysis technique will be further discussed later on, in Section III-B.2.

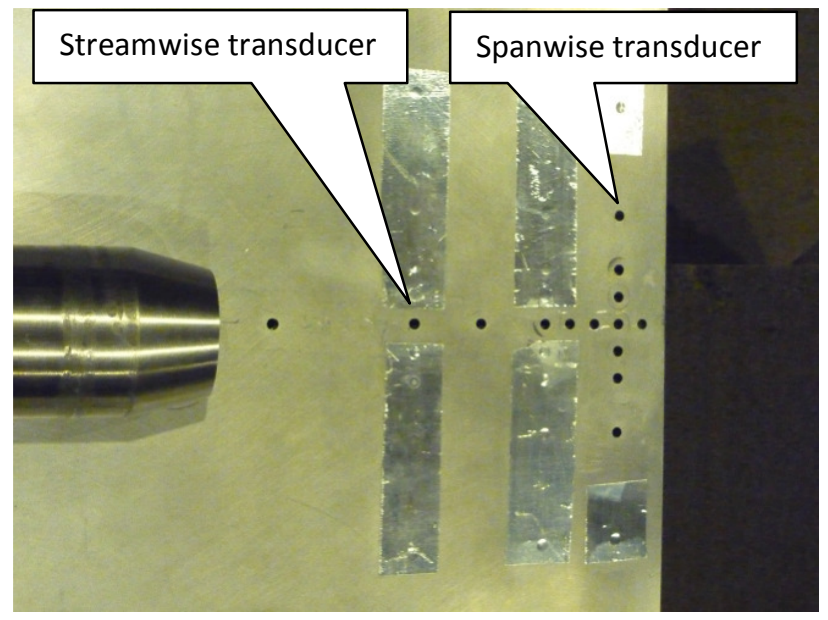

Figure 4. Doak laboratory near-field surface pressure transducer T-array

\section{Results}

\section{A. Far-Field Analysis}

Typically, when characterizing a broadband jet noise source, the temptation, and certainly the historical convention, is to display spectral data in $1 / 3^{\text {rd }}$ octave bands. For this study, however, it is necessary to focus on much finer bands of low frequency noise in order to find the true source peak and then to track its behaviour. Thus, many of the following spectral plots contain constant bandwidth data, where $\Delta f=400 \mathrm{~Hz}$. This particular bandwidth value has also been chosen for illustrative purposes since it is easier to view more than one data series on a single plot compared to standard $10 \mathrm{~Hz}$ narrowband bandwidth data.

\section{Interaction source peak frequency}

As a first look at the data, Figure 5 shows that the peak of the low frequency interaction source increases with velocity, as expected. Looking at the $\triangle S P L$ between the isolated and installed cases, it is also clear that the source strength has a weaker dependence on velocity compared to the isolated jet mixing noise source.

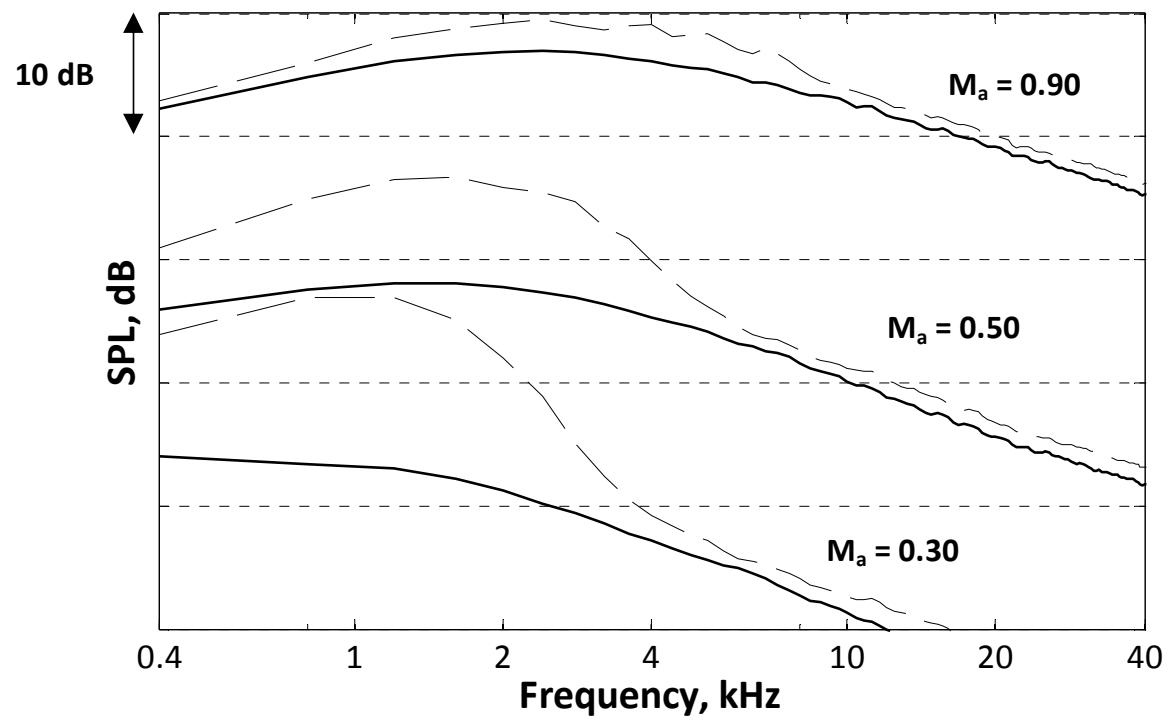

Figure 5. Isolated jet (solid lines) versus installed jet (broken lines) interaction source velocity dependence, $\mathrm{H} / \mathrm{D}=\mathbf{0 . 6 7}, \mathrm{L} / \mathrm{D}=2$ 
Extending the shield further downstream from the nozzle exit moves the interaction peak to a lower frequency (see Figure 6a). As the TE is situated further downstream from the nozzle, larger eddies within the jet's upper free shear layer begin to dominate the near-field. These larger scales inherently generate lower frequency hydrodynamic noise, which is radiated from the TE to the far-field. These eddies (or regions of turbulence over which fluctuations of velocity are highly correlated) also contain more energy than eddies generated upstream, which accounts for the increase in interaction noise strength above the isolated jet case.
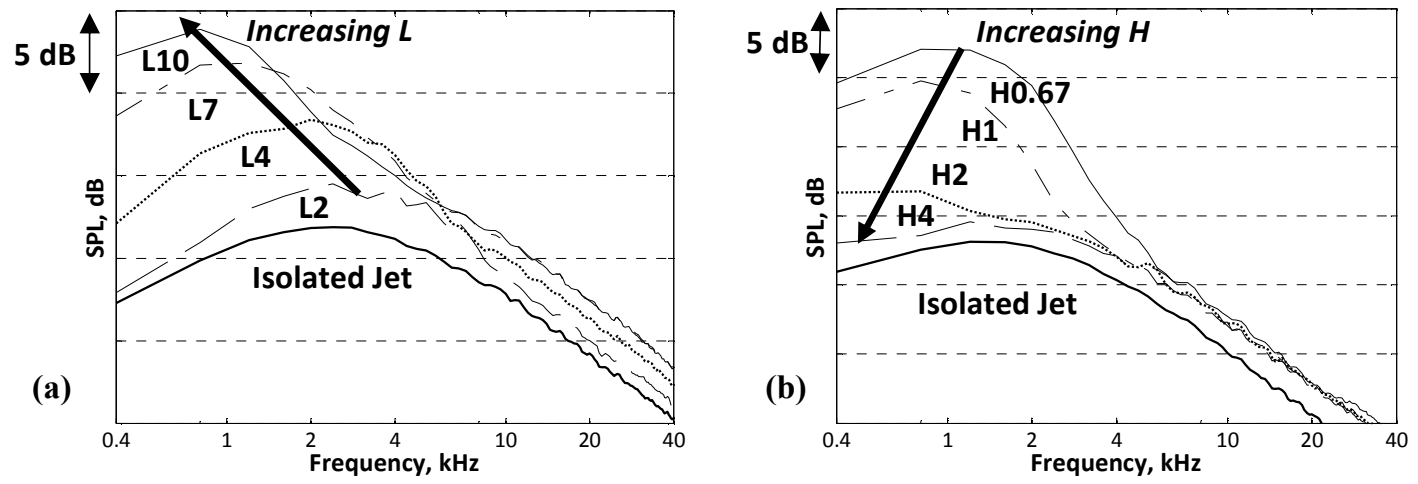

Figure 6. Installed dipole peak frequency (a) $L$ dependence $(H / D=0.67)$ and

(b) $H$ dependence $(\mathrm{L} / \mathrm{D}=4) ; \theta=90^{\circ}, \varphi=0^{\circ}$ (unshielded)

Figure $6 \mathrm{~b}$ shows that the interaction peak frequency also increases when the TE is brought closer to the jet. This result has been noted in previous near-field isolated jet studies and relates to the additional contribution of the hydrodynamic near-field ${ }^{9-11}$. More specifically, near-field pressure fluctuations have been found to decay proportionally to $k r^{-2}, k r^{-6.67}$ and $k r^{-7 / 3}$ depending upon whether they lie within the jet's acoustic, linear hydrodynamic or non-linear hydrodynamic regions, respectively ${ }^{9}$. Arndt et al. (1996) concluded that $k r=2$ is the frequency dependent dividing line between the near- and far-fields of a turbulent jet. In other words, when $k r>2$, the evanescent hydrodynamic near-field strength becomes negligible and the jet acoustic field radiates alone to the far-field as per geometrical acoustics (i.e. $k r^{-2}$ ). Further discussion of these field decay trends can be found in Section III-B-1.

In order to collapse, and hence to scale, the installed dipole spectrum peaks, the most representative characteristic length-scale and velocity of the interaction physics should be chosen. Since it has been shown that the peak is dependent on both $H$ and $L$, no single Strouhal relationship can exist based upon one universal geometrical wing parameter. Thus, initially, the nozzle diameter $D$ is used as the characteristic length-scale. Both the exit jet velocity $U_{j}$, and the convection velocity $U_{c}$ of the pressure field passing the TE were investigated. It was found that the more suitable collapse was given, however, using $U_{j}$ (see Figure 7), which is consistent with Head and Fisher ${ }^{4}$. Essentially, this tells us that the interaction source is primarily a function of jet power rather than turbulent boundary layer surface pressure fluctuation.
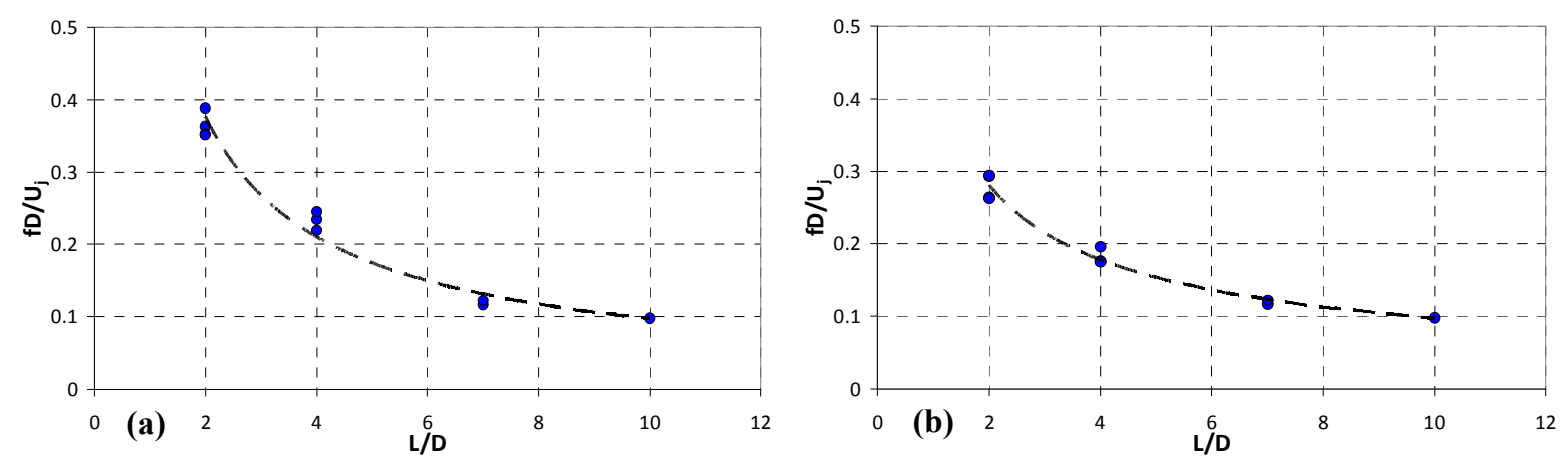

Figure 7. Installed interaction source peak frequency location $S t_{D}$ as a function of shield length $L / D$ for (a) $H / D=0.67$ and (b) $H / D=1$ 
Figure 7 also illustrates that more than one single source generation mechanism exists. At large $L$, the peak frequencies collapse both for $H / D=0.67$ and $H / D=1$. This would suggest that, for $S t_{D}<0.1$, the TE is physically wetted by the flow (i.e. located within the non-linear, rotational hydrodynamic region of the jet) and, thus, is seen as an acoustically compact surface compared to the wavelength of sound generated from the eddy within the shear layer. Such configurations, therefore, are expected to follow Curle's $U^{6}$ radiated sound intensity theory for a fluctuating dipole surface. However, when the TE lies within the linear, evanescent hydrodynamic field of the jet, or when the wavelength of sound produced from an 'eddy' is comparable to its distance from the TE, the strength and frequency content of the interaction source becomes extremely radially sensitive. Finally, when an eddy is sufficiently remote from the edge, the convecting turbulence is essentially unbounded and no interaction exists. Ffowcs-Williams and Hall (1970) assigned inequalities to represent two of these three scenarios. Eddies which satisfy $\left(k \bar{r}_{0}\right)^{1 / 2}>>1$, where $\bar{r}_{0}$ is the distance of the centre of an eddy from the edge, have the sound output of the Lighthill quadrupoles associated with an eddy in free space and propagate unaffected by the edge as would be predicted by geometrical acoustics such that,

$$
I \propto \rho U_{j}^{3}\left(\frac{U_{j}}{a_{0}}\right)^{5} \frac{\delta^{2}}{R^{2}}
$$

Eddies which satisfy $2 k \bar{r}_{0}<<1$, however, introduce an additional factor $\left(k \bar{r}_{0}\right)^{-3}$ associated with the quadrupole fluid motion in a plane normal to the edge such that,

$$
I \propto \rho U_{j}^{3}\left(\frac{U_{j}}{a_{0}}\right)^{2} \frac{\delta^{2}}{R^{2}}
$$

The following sections will illustrate the velocity dependence of the above scenarios together with their associated directivity.

\section{Interaction source strength}

It is possible to infer more detail about the different types of sources present by comparing the behaviour of the radiated energy at $\theta=90^{\circ}$ with acoustic jet Mach number. The overall frequency-integrated sound pressure level (OASPL) is used to represent this radiated energy and the isolated jet noise is subtracted from each installed case so that no corruption from the quadrupole jet mixing noise is present. Figure 8 shows how the isolated jet $U_{j}^{8}$ acoustic quadrupole mixing source (the solid line) gives way to a $U_{j}^{6}$-type trend at large values of $L$ (the star-marked dashed line). This is also the case at low velocities (i.e. $M_{a} \leq 0.75$ ) as the plate TE is 'wetted' by the convecting eddies within the jet's non-linear hydrodynamic field. Then, as the TE is moved upstream and as the relative distance between eddy size and TE increases, the interaction source begins to radiate more proportionally to $U_{j}^{5}$ (the circlemarked dashed line). The isolated $U_{j}^{8}$ and installed $U_{j}^{6}$ dependencies are consistent with Lighthill and with both Curle $^{1}$ and Head and Fisher's ${ }^{4}$ findings for acoustically compact surfaces. The $U_{j}^{5}$ dependency for the diffracted hydrodynamic field is also consistent with Ffowcs-Williams and Hall ${ }^{2}$, however, there exists an intermediate mechanism for the $L / D=4$ case, which goes more like $U_{j}^{5.5}$ (the triangle-marked dashed line). This transition between the $U_{j}^{6}$ and $U_{j}^{5}$ power laws is a new result and its understanding requires further research into the behaviour of the linear hydrodynamic near-field.

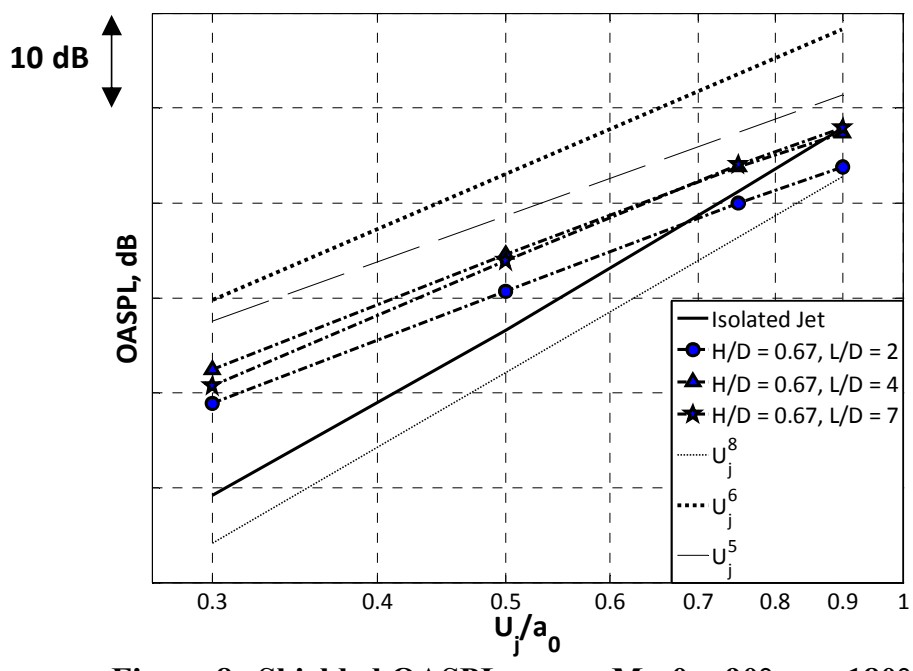

Figure 8. Shielded OASPL versus $M_{a}, \theta=90^{\circ}, \varphi=180^{\circ}$

American Institute of Aeronautics and Astronautics 
It is clear that, at low velocities, the non-linear, rotational hydrodynamic field (or dipole) source mechanism is weaker than noise generated by diffraction of the linear hydrodynamic field. The rotational field acts as a weaker radiating source essentially because the component of the fluctuating turbulent forces (from eddies), which act perpendicular to the TE, are not as ordered as the linear convecting hydrodynamic field.

\section{Interaction source directivity}

When compared to past isolated jet directivity studies ${ }^{12}$, the unmarked solid and unmarked broken lines in Figure 9a show a consistent trend towards typical far-field jet peak behavior ${ }^{12}$. It is difficult, however, to validate the complete jet directivity patterns, in this study, due to the lack of extreme polar angle data. The installed minus isolated jet directivity, by comparison to the isolated jet, shows a much more omnidirectional pattern on the unshielded side of the jet (the square-marked solid line). Interestingly, a significant amount of additional energy (up to $8 \mathrm{~dB}$ ) is seen to propagate into the forward jet arc here (i.e. for $\theta>90^{\circ}$ ). The shielded installed data (the circlemarked dashed line in Figure 9a), however, depicts a much more typical $\sin \theta$ dipole pattern, which is consistent with Curle's formulation ${ }^{1}$. This asymmetry in directivity is, at least partly, seen because the shielded side of the jet sees a clear propagation path whereas the unshielded interaction noise generated at the TE not only has to propagate through the turbulent jet plume but also will contain reflections from the plate. Further installed jet forward arc measurement is planned to address this particular directivity conundrum.
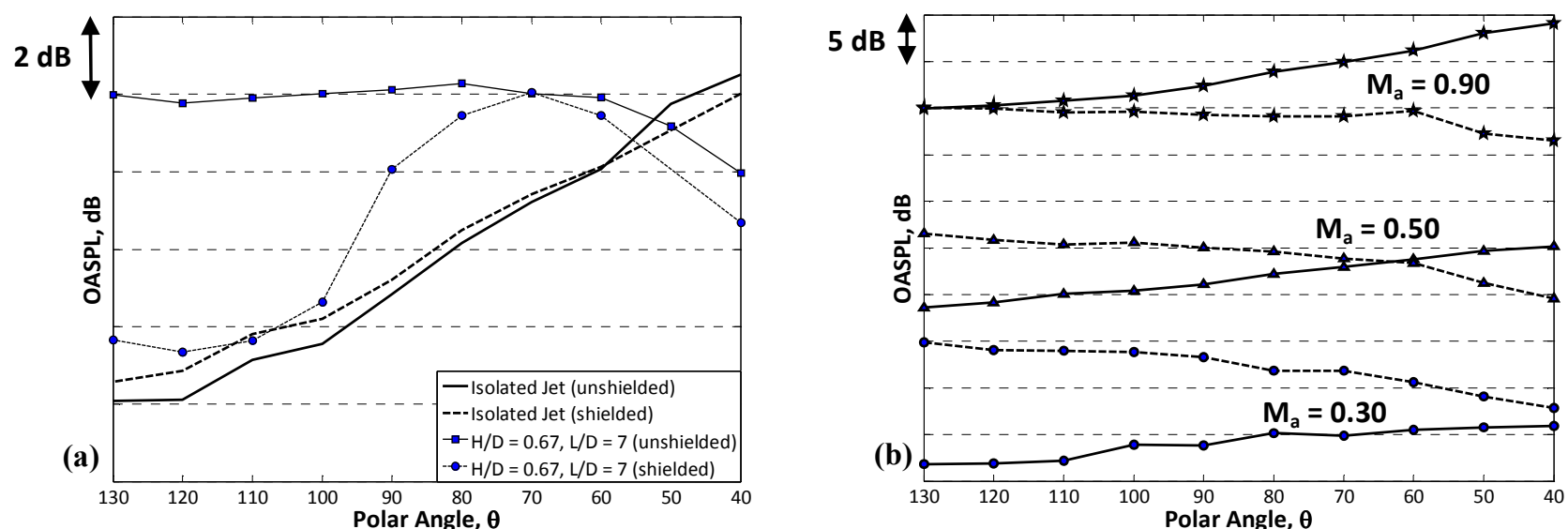

Figure 9. (a) Polar OASPL directivity isolated jet vs. (installed jet - isolated jet) and unshielded $\left(\varphi=0^{\circ}\right)$ vs. shielded $\left(\varphi=180^{\circ}\right) ; M_{a}=0.75$ and (b) polar unshielded OASPL directivity isolated jet [solid lines] vs. (installed jet - isolated jet) [broken lines] vs. acoustic Mach number; H/D = 0.67, $\mathrm{L} / \mathrm{D}=2$

Figure $9 \mathrm{~b}$ shows that the interaction noise directivity pattern (the broken lines) remains constant with increasing flow velocity. The delta between the interaction noise and the isolated jet noise in the forward arc, however, decreases with increasing jet velocity until the $U_{j}^{8}$ quadrupole mixing noise dominates all polar angles $\left(\right.$ at $\left.M_{a}=0.90\right)$.

The azimuthal dipolar directivity can be seen in Figure 10, which is consistent with Head \& Fisher ${ }^{4}$. The dipole is accentuated as the turbulence intensity at the TE is increased (i.e. as $L$ is increased). As with the polar directivity, the interaction noise azimuthal directivity is independent of jet velocity. 


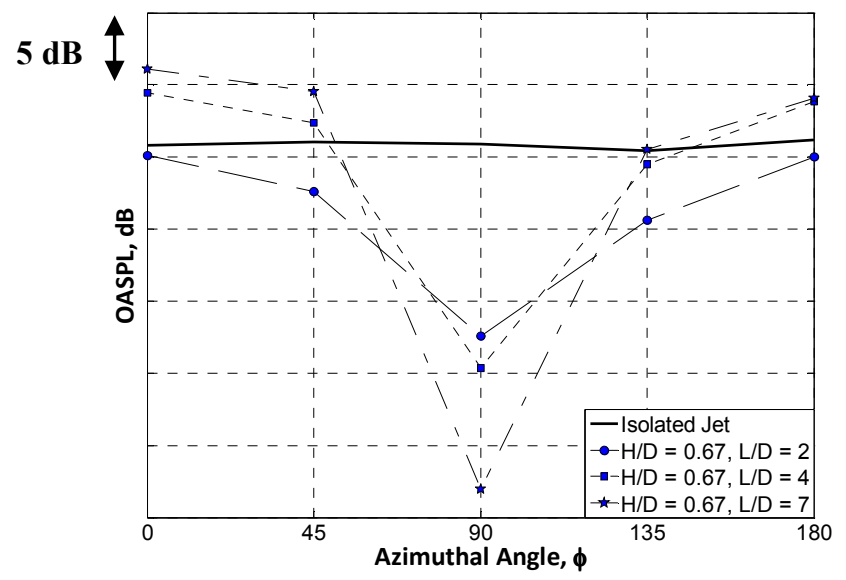

Figure 10. Azimuthal OASPL directivity isolated jet vs. (installed - isolated jet); $\theta=0^{\circ}, M_{a}=0.75$

\section{B. Near-Field Analysis}

1. Near-field versus far-field correlation

In addition to Head and Fisher's findings, the first important point to make is that a one-to-one frequency correspondence between near- and far-field interaction noise peaks does not always exist. This is particularly relevant when a high-speed $\left(M_{a}>0.5\right)$, subsonic jet is closely-coupled $(H<2 D)$ to the plate. It is believed that this is because the interaction noise becomes contaminated by the much stronger $U_{j}^{8}$ acoustic field produced by the jet mixing region. Further experimental investigation is planned to model the frequency content of the linear jet hydrodynamic near-field in order to validate this frequency mismatch hypothesis. The following near-to-far-field coherence graphs illustrate the deterioration of this one-to-one correspondence. Coherence decreases both with Mach number (Figure 11a) and with the level of interaction between flow and solid boundary (e.g. increasing shield length, $L$ - Figure $11 \mathrm{~b})$.
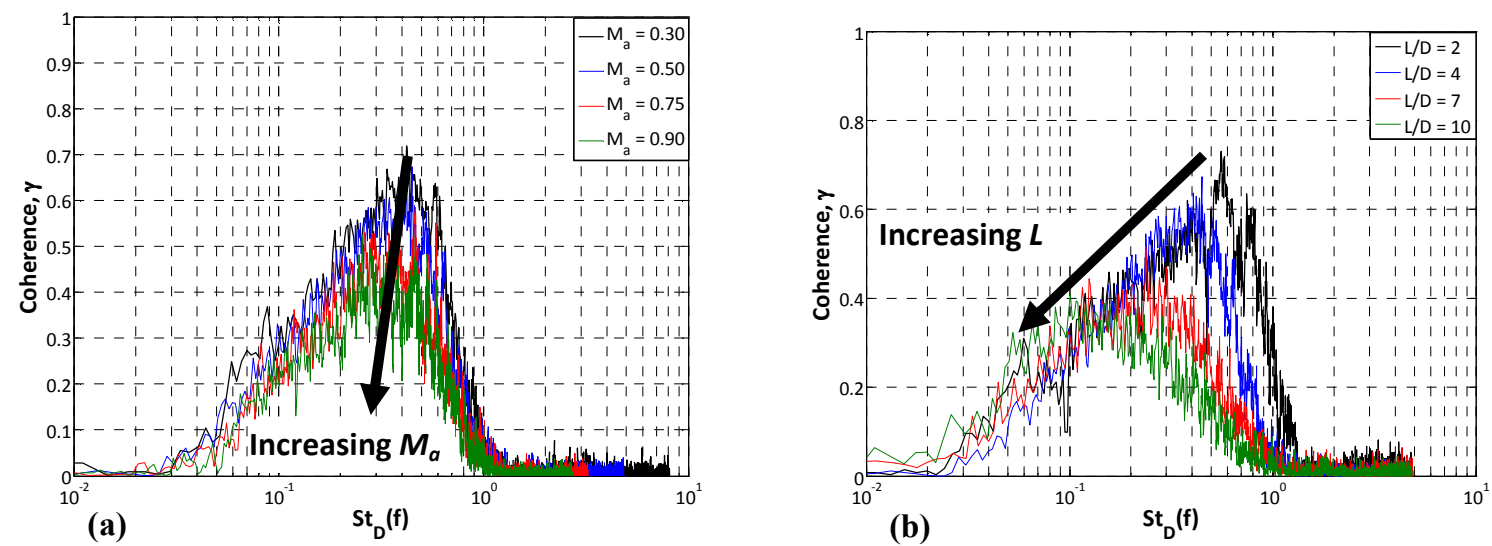

Figure 11. Near-field to far-field coherence plots (a) vs. acoustic Mach number, $M_{a}$ and (b) vs. $L$

\section{Calculation of $U_{c}$}

Analysis of the phase relationships between the five equally-spaced, streamwise near-field surface pressure transducers closest to the plate's trailing edge can serve to give an understanding of the convection velocities of the pressure fields as they pass along the plate. The constraints involved with such a technique include the streamwise wavenumber resolution $\Delta k_{1}$ - a function of the total length of the array - and the coherence between the first and last transducer, which, essentially, governs the smallest measurable length-scale that can be tracked traveling across the transducers. Since the phase information within a single eddy is assumed to be coherent, when coherence is lost, the transducers effectively pick up neighbouring eddies, and, thus, the convection velocity is incalculable. 
Assuming good phase and coherence over the array, the spatial integral of the cross-spectral densities between the streamwise surface pressure near-field microphones is taken. This technique breaks the signal down into the acoustic and hydrodynamic wavenumber components of the convecting pressure field. This streamwise wavenumber-frequency $\Pi_{p_{i} p_{j}}\left(k_{1}, \omega\right)$ relationship can be expressed mathematically as,

$$
\Pi_{p_{i} p_{j}}\left(k_{1}, \omega\right)=\int_{-\infty}^{\infty} \Phi_{p_{i} p_{j}}\left(\varepsilon_{1}, \omega\right) e^{-i k_{1} \varepsilon_{1}} d \varepsilon_{1}
$$

where $\Phi_{p_{i} p_{j}}$ is a matrix of constant bandwidth cross-spectral densities, and $\varepsilon_{1}$ is the constant streamwise transducer separation. As has already been discussed, the spacing between the transducers essentially dictates the minimum size of the eddy possible to track across the plate (i.e. the high frequency acoustic cut-off) and the total length of the array defines the wavenumber resolution. Figure 12, for example, depicts a heavily wetted case, where the majority of the pressure convecting across the plate TE is the subsonic, irrotational hydrodynamic field.

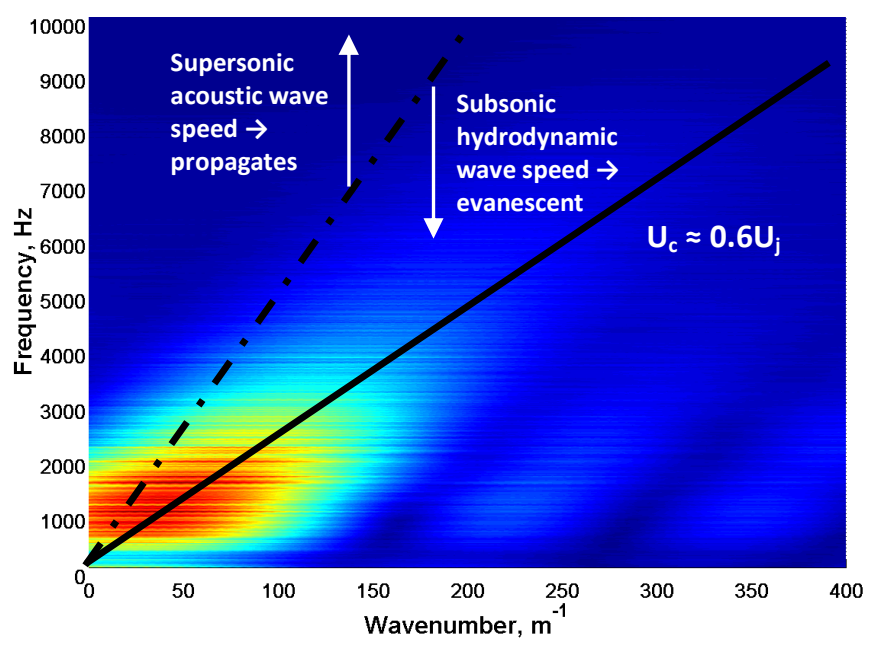

Figure 12. Wavenumber-frequency plot of near-field pressure $-\mathrm{H} / \mathrm{D}=\mathbf{0 . 6 7}, \mathrm{L} / \mathrm{D}=10, \mathrm{M}_{\mathrm{a}}=\mathbf{0 . 9}$ [dotted line - speed of sound; solid line - convection velocity of pressure field]

As has been demonstrated by several previous studies, the value of $U_{c}$ is a frequency dependent quantity. Figure 13 shows three jet/plate configurations. When the jet/plate separation $H / D$ is greater than 1 , the convecting pressure field over the plate TE becomes supersonic. The frequency at which this velocity $U_{c} / U_{j}$ exceeds unity will give some idea as to the location of the edge of the linear hydrodynamic field for different parts of the jet and, thus, will provide a frequency-dependent cut-off radial distance for interaction noise generation. The trade-off here, however, is transducer sensitivity versus the radial decay rate of the linear hydrodynamic field and so must be treated with caution. This is to be the subject of future experimental research.

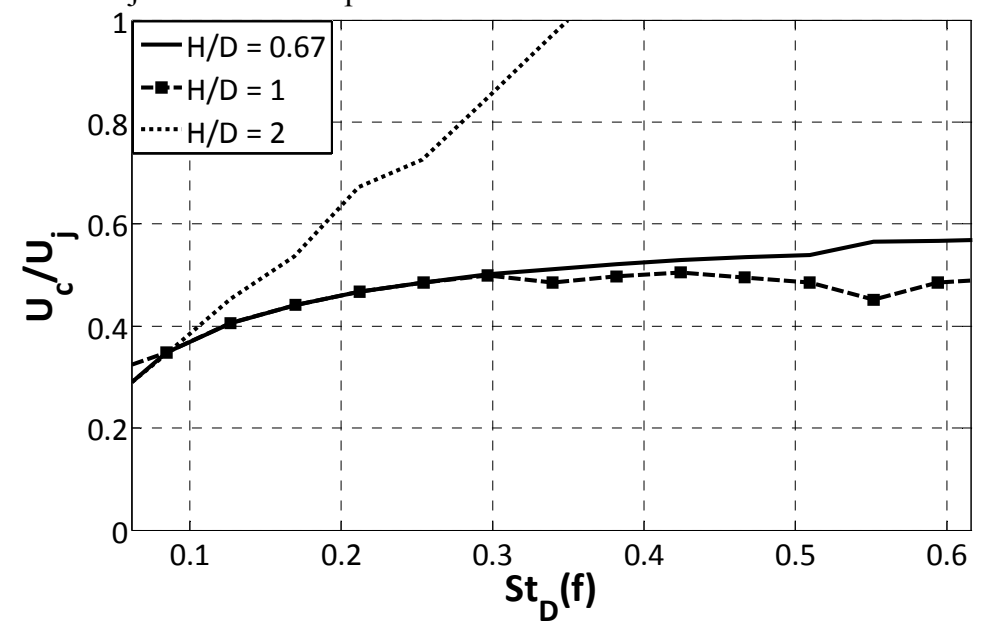

Figure 13. Convection velocity plot illustrating hydrodynamic vs. acoustic

American Institute of Aeronautics and Astronautics 


\section{Comparison with Amiet}

Presented here is a comparison of Amiet's model with the measured far-field acoustic data. According to Amiet's model for trailing edge noise ${ }^{8}$, the far-field power spectral density (PSD) can be obtained from,

$$
S_{p p}(\boldsymbol{x}, \omega)=\left(\frac{\omega x_{3} b}{2 \pi c S_{0}^{2}}\right)^{2} 2 \pi L_{s}\left|I_{r}\left(\frac{\bar{\omega}}{U_{c}}, \bar{k} \frac{x_{2}}{S_{0}}\right)\right|^{2} \Pi_{0}\left(\frac{\omega}{U_{c}}, k \frac{x_{2}}{S_{0}}\right)
$$

where $I_{r}$ is the radiation integral (given in Ref. 8) and $\Pi_{0}$ denotes the wavenumber spectral density of the incident gust with amplitude $A_{0}$. The wavenumber spectral density $\Pi_{0}$ represents the energy of the incident wall pressure fluctuations at frequency $\omega$ for a given spanwise wavenumber, and is given by,

$$
\Pi_{0}\left(\frac{\omega}{U_{c}}, k \frac{x_{2}}{S_{0}}\right)=\frac{1}{\pi} \Phi_{p p}(\omega) l_{y}\left(k \frac{x_{2}}{S_{0}}, \omega\right)
$$

In this factorization, $\Phi_{p p}$ is the wall pressure spectrum corresponding to the incident aerodynamic fluctuations, measured using the flush surface-mounted pressure sensors along the trailing edge of the plate. The following simple model has also been used for modeling the correlation length,

$$
l_{y}\left(k_{2}, \omega\right)=\frac{\omega /\left(b_{c} U_{c}\right)}{k_{2}^{2}+\omega^{2} /\left(b_{c} U_{c}\right)^{2}}
$$

Figure 14, below, shows the current agreement between Amiet and measured far-field data at $\theta=90$ and at different Mach numbers.

(a)

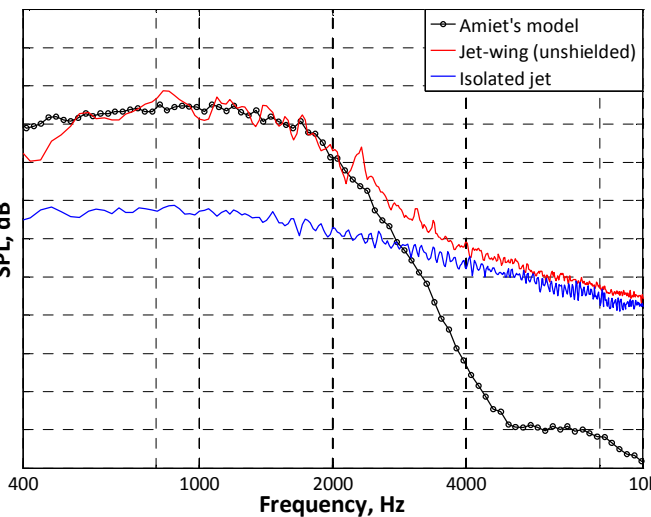

(c)

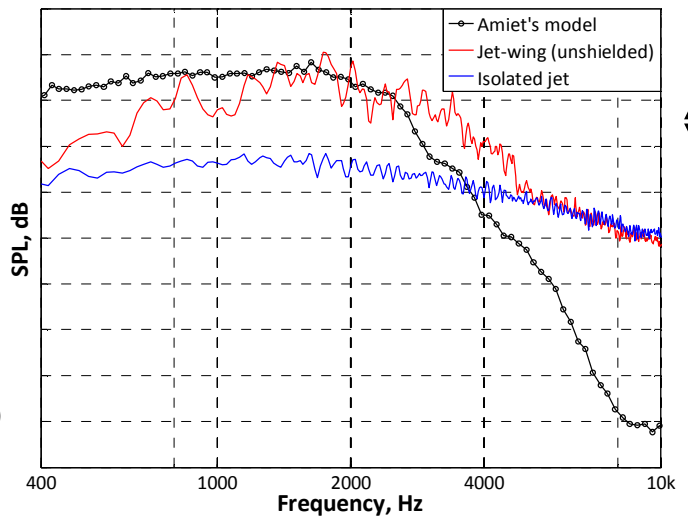

$\checkmark 5 \mathrm{~dB}$

(b)
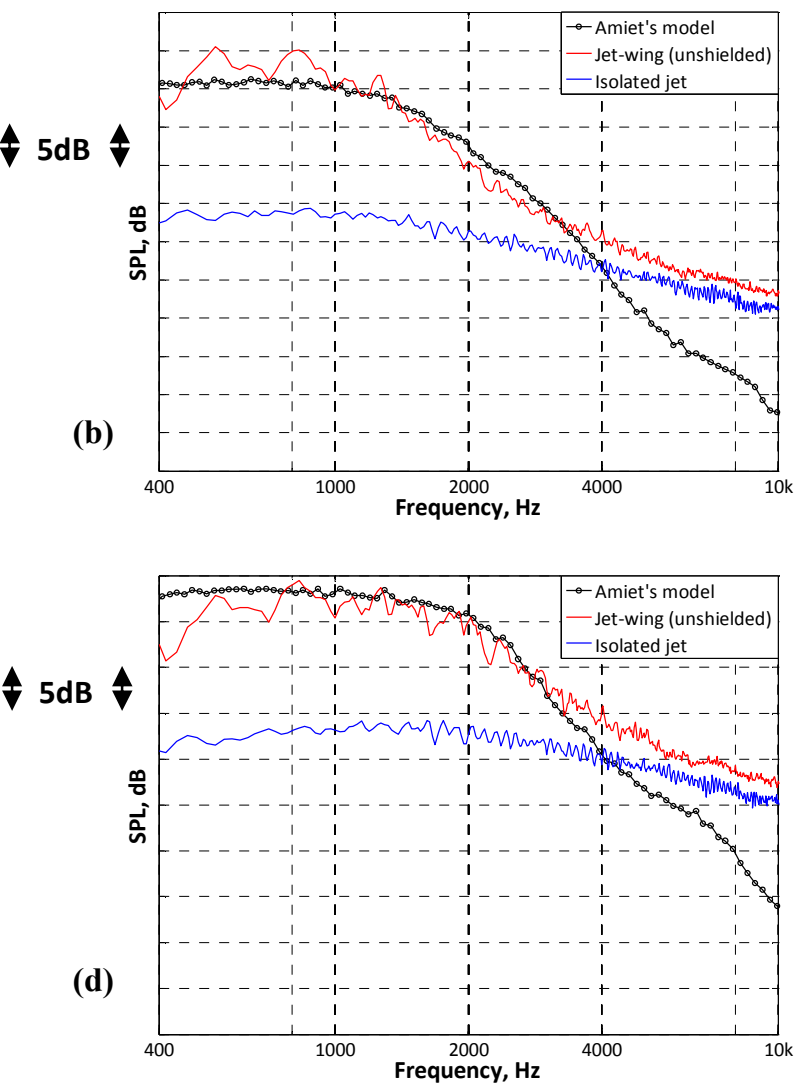

Figure 14. Amiet prediction results (a) $\mathrm{H} / \mathrm{D}=0.67, \mathrm{~L} / \mathrm{D}=2, \mathrm{M}_{\mathrm{a}}=0.3$, (b) $\mathrm{H} / \mathrm{D}=0.67, \mathrm{~L} / \mathrm{D}=4, \mathrm{M}_{\mathrm{a}}=0.3$, (c) $\mathrm{H} / \mathrm{D}=0.67, \mathrm{~L} / \mathrm{D}=2, \mathrm{M}_{\mathrm{a}}=0.5$ and (d) $\mathrm{H} / \mathrm{D}=0.67, \mathrm{~L} / \mathrm{D}=4, \mathrm{M}_{\mathrm{a}}=0.5$ 


\section{Conclusion}

An extensive experimental investigation into the interaction between a subsonic jet and a flat plate has been conducted. Both near- and far-field measurements were made in order to better understand the source generation mechanisms involved in close-coupled jets. It was discovered that the radial location of the plate TE within specific parts of the jet hydrodynamic field is the key to determining the noise generating mechanisms and, hence, the correct scaling law. If located within the non-linear, rotational hydrodynamic region (i.e. for $\mathrm{kr}^{-7 / 3}$ ), the interaction noise intensity scales classically as per a fluctuating edge dipole $-I \propto U^{n}$, where $n=6$. However, if the TE is situated within the linear, irrotational hydrodynamic region (i.e. for $k r^{-6.67}$ ), interaction noise will be generated through diffraction of this field and $n$ will take a value between $5 \leq n<6$, depending on the precise radial location of the TE. As the radial distance between source and TE increases, within the linear hydrodynamic region, the radiated energy dependence on velocity will become weaker. Questions were also raised concerning the forward arc interaction noise directivity patterns produced on the unshielded side of the installed jet. It has become apparent that more extreme forward arc jet angle data is required to help address this.

It was also found that the strength of the jet acoustic mixing noise, particularly at high Mach numbers, is responsible for shifting the hydrodynamic near-field signal peak to a higher frequency compared to the far-field noise peak. Further near-field acoustic experiments, however, are planned in order to help model the strength, frequency content and decay of this linear, hydrodynamic jet field when installed beneath a solid body.

Finally, successful implementation of Amiet's trailing edge far-field noise model has been performed for low Mach number jets using near-field point spectra close to the plate TE. Future more accurate separation of the jet's hydrodynamic near-fields from the purely acoustic near-field is expected to reveal more accurate interaction noise predictions for higher velocity subsonic jets.

\section{Acknowledgments}

The authors would like to thank the Technology Strategy Board (TSB), the Engineering and Physical Sciences Research Council (EPSRC) and QinetiQ Ltd. (QQ) for providing the funding necessary to carry out this research. The authors would also like to thank R. A. Pinker for his invaluable expertise during the experiment design phase.

\section{References}

${ }^{1}$ Curle, N, "The influence of solid boundaries upon aerodynamic sound", Proceedings of the Royal Society of London, 231A, pp. $505-517,1955$

${ }^{2}$ Ffowcs-Williams, J. E. and Hall, L. H., "Aerodynamic sound generation by turbulent flow in the vicinity of a scattering half plane”, Journal of Fluid Mechanics, Vol. 40, Pt. 4, pp. 657-670, 1970

${ }^{3}$ Crighton, D. G. and Leppington, F. G., "Scattering of aerodynamic noise by a semi-infinite compliant plate", Journal of Fluid Mechanics, Vol. 43, Part 4, pp. 721-736, 1970

${ }^{4}$ Head, R. W., and Fisher, M. J., "Jet/Surface Interaction Noise: - Analysis of Far-Field Low Frequency Augmentations of Jet Noise due to the Presence of a Solid Shield", $3^{\text {rd }}$ AIAA Aeroacoustics Conference, No. 502, 1976

${ }^{5}$ Chase, D. M., "Noise Radiated from an Edge in Turbulent Flow", AIAA Journal, Vol. 13, No. 8, pp. 1041-1047, 1975.

${ }^{6}$ Meecham, W. C., Bui, T. D. and Miller, W. R., "Diffraction of sound by the edge of a rigid baffle", Journal of the Acoustical Society of America, Vol. 70, No. 5, pp. 1531-1533, 1982

${ }^{7}$ Miller, W. R., "Flight effects for jet-airframe interaction noise", AIAA Journal, Vol. 83, No. 0784, 1983

${ }^{8}$ Amiet, R. K., "Noise due to turbulent flow past a trailing edge", Journal of Sound and Vibration, Vol. 47, No. 3, pp. $387-$ 393, 1976

${ }^{9}$ Arndt, R. E. A., Long, D. F. and Glauser, M. N., “The proper orthogonal decomposition of pressure fluctuations surrounding a turbulent jet", Journal of Fluid Mechanics, Vol. 340, pp. 1-33, 1997

${ }^{10}$ Harper-Bourne, M., "On Modelling the Hydrodynamic Field of High-Speed Jets", $10^{\text {th }}$ AIAA/CEAS Aeroacoustics Conference, No. 2830, 2004

${ }^{11}$ Tinney, C. E. and Jordan, P., "The near pressure field of co-axial subsonic jets”, Journal of Fluid Mechanics, Vol. 611, pp. 175-204, 2008

${ }^{12}$ Hubbard, H. H., "Aeroacoustic of Flight Vehicles: Theory and Practice - Volume 1: Noise Sources”, NASA Technical Report 90-3052, Vol. 1, pp. 224, 1991. 